\title{
Maternal anxiety associated with fetal echocardiography
}

\author{
Alexandra Channing ${ }^{1}$, Katherine Rosenberg ${ }^{1}$, Catherine Monk ${ }^{2}$, Charles S. Kleinman ${ }^{1}$, \\ Julie S. Glickstein ${ }^{1}$, Stephanie M. Levasseur ${ }^{1}$, Lynn L. Simpson ${ }^{3}$, Ismee A. Williams ${ }^{1 *}$ \\ ${ }^{1}$ Department of Pediatrics, Division of Pediatric Cardiology, College of Physicians \& Surgeons, Columbia University, Morgan Stanley \\ Children's Hospital of New York, New York, USA \\ ${ }^{2}$ Department of Psychiatry, Behavioral Medicine, Columbia University Medical Center, New York, USA \\ ${ }^{3}$ Department of Obstetrics \& Gynecology, Division of Maternal Fetal Medicine, College of Physicians \& Surgeons, Columbia University, \\ Sloane Hospital for Women, New York, USA \\ Email: iib6@,columbia.edu
}

Received 5 November 2011; revised 10 March 2012; accepted 22 March 2012

\begin{abstract}
Background: Women awaiting fetal echocardiography (fECHO) report high anxiety. It is unclear if anxiety decreases after performance of fECHO. Methods: At fECHO, subjects' current (state) vs baseline (trait) anxiety was assessed using the Spielberger State-Trait Anxiety Inventory. Anxiety scores of the pre- and post-fECHO groups were compared. Results: From January 2007 to January 2009, we recruited 84 subjects: 40 pre-fECHO and 44 post-fECHO. Of the post-fECHO group, 30 had normal fetal cardiac structure and function confirmed, 12 were told of an abnormality, and 2 were told to follow up equivocal results. Anxiety scores were compared between the 40 pre-fECHO subjects and the 30 post-fECHO subjects with normal results. The mean state anxiety score of the pre-fECHO group was higher than that of the post-fECHO group $(42.1 \pm 15.1$ vs $30.8 \pm 8.5, p<$ 0.001); there was no difference in trait scores. Neither state nor trait anxiety was associated with maternal age, parity, history of miscarriage or known fetal anomaly. Compared to those with a normal fECHO $(\mathrm{N}=30)$, subjects with an abnormal fECHO result ( $N$ $=12)$ had higher state anxiety $(46.8 \pm 15.5$ vs $30.8 \pm$ 8.5, $\mathrm{p}=0.005)$. There was no difference in anxiety scores between subjects awaiting fECHO and postfECHO subjects who had an abnormal result. Conclusion: Immediately following normal fECHO, women report low anxiety compared with women awaiting fECHO. Women awaiting fECHO report anxiety levels that are as high as women who are told there is fetal cardiac anomaly.
\end{abstract}

Keywords: Maternal Anxiety; Prenatal Testing; Birth Defects; Fetal Echocardiography

"Corresponding author.

\section{INTRODUCTION}

The association between maternal anxiety and prenatal testing is well documented. Studies report women with high anxiety levels prior to and following both non-invasive testing, such as routine obstetrical ultrasound, and invasive testing, such as chorionic villus sampling and amniocentesis [1-6]. Cristofalo et al. found that women expressed feelings of fear, shock and decreased attachment in response to the prenatal detection of an isolated choroid plexus cyst, a benign structural variant, despite reassuring counseling from providers [7]. Furthermore, maternal anxiety is associated with adverse maternal and fetal effects, including preterm labor, preeclampsia, spontaneous abortion, and congenital malformations in both animal and human studies [8-12]. Finally, changes in health care delivery and physician and patient expectations have increased interest in integrated medical care designed to address not just physical health but also mental health. All of these factors support the need to address additional attention to maternal psychosocial and emotional well-being during pregnancy-related medical procedures.

The indications for referral for fetal echocardiography (fECHO), or targeted ultrasound screening of the fetal heart, have increased over recent years. Current guidelines state that women should be referred not just when there is a suspicion of a cardiac anomaly or when the fetal heart is not well visualized on an obstetric anatomy scan, but also in the setting of an abnormal first trimester screen, a history of maternal diabetes or a family member with congenital heart disease, in vitro fertilization, or presence of multiple gestations among others [13]. Though the true prevalence of fECHO referral among pregnant women has not been reported in recent years, it has been our experience that given the change in guidelines and the advancements in obstetric ultrasound screening, more women are undergoing fECHO today than in prior years. At our institution alone, the number of fetal echocardio- 
grams performed has more than doubled over the past 5 years from around 500 in 2005 to well over 1200 in 2010.

We previously reported that women presenting for fECHO experience high anxiety compared with pregnant women not presenting for fECHO [14]. Whether anxiety remains high following the scan and physician counseling remains unknown. The purpose of this study was to compare the anxiety scores of women awaiting $\mathrm{fECHO}$ with those of women who had just undergone a $\mathrm{fECHO}$. Furthermore, we sought to evaluate how the fECHO result would influence maternal anxiety among women who had completed the fetal cardiac assessment.

\section{METHODS}

\subsection{Population}

From January 2007 to January 2009, we conducted an IRB-approved cross-sectional study of pregnant women presenting for fECHO at the Morgan Stanley Children's Hospital of New York. All women presenting for an initial fECHO were eligible. Women unable to read English were excluded. All participants provided written informed consent prior to entering the study.

Two groups of women were recruited: one group was awaiting a fECHO (pre-fECHO) while the other group had just completed the scan and had discussed the results with the physician (post-fECHO). (Figure 1) In the postfECHO group, the study was introduced following the scan and counseling session. Due to standard psychological testing limitations, individuals were not tested repeatedly pre- and post-fECHO as this would lead to inaccurate results secondary to test-retest recall bias. After obtaining informed consent, the Spielberger StateTrait Anxiety Inventory Form Y (STAI) was administered in a standardized fashion by a research assistant [15]. Subjects were also given the self-administered Pregnancy Stress Scale, developed by the investigators for the purposes of this study, which recorded pregnancy specific concerns, demographic and medical information [14].

The STAI is a validated measure of self-reported anxiety that differentiates between anxiety related to the baseline personality of the individual (trait) and anxiety that is related to a current external experience (state). The tool consists of 40 statements: 20 designed to assess trait anxiety, measuring how the subject "generally feels" and 20 designed to assess state anxiety, measuring how the subject feels "right now". Scores for both trait and state anxiety can range from 20 to 80 and higher scores indicate greater anxiety. Reported average STAI scores among pregnant women who were screened as negative for clinical depression, anxiety disorder, and other psychiatric co-morbidities were $34.1 \pm 9.5$ for trait and 30.7 \pm 8.8 for state, and among women with documented anxiety disorders was $40.1 \pm 8.7$ for trait and $35.9 \pm 9.3$ for state [16]. Field et al. surveyed 166 pregnant women and concluded that a trait score higher than 38 was considered to be highly anxious [17].

Results from the STAI questionnaire were not disclosed to subjects. All study subjects, regardless of their anxiety levels, received the contact information of a perinatal psychologist (CM).

\subsection{Statistical Analysis}

Basic descriptive characteristics were analyzed using means and standard deviations for normally distributed data. Baseline differences between the pre-fECHO and post-fECHO groups were assessed using a Student's t test and two-by-two tables with the Chi Square test statistic or the Fisher's Exact test statistic when appropriate. To compare the STAI anxiety scores between the preand post-fECHO groups, an independent Student's t test was conducted. Student's t tests and ANOVA were used to ascertain differences in anxiety between those who had a history of miscarriage, were primigravid, were of advanced maternal age and were referred with fetal cardiac versus extra-cardiac anomaly. Using multivariate regression analysis, we evaluated the difference in state scores among pre- and post-fECHO subjects while controlling for other possible confounders. Entry criterion for the multivariable analysis was set at 0.1. All other alpha values were set at 0.05 .

\section{RESULTS}

Of the 157 women eligible for the study, 84 successfully completed the study questionnaires, 40 prior to the $\mathrm{fECHO}$ and 44 after the fECHO. Of the 44 post-fECHO subjects, 30 had normal results (normal post-fECHO group), 12 had abnormal results (abnormal post-fECHO group), and 2 were scheduled for follow up fetal echocardiograms to assess potential abnormalities. We limited our initial
Group 1: Pre-fECHO

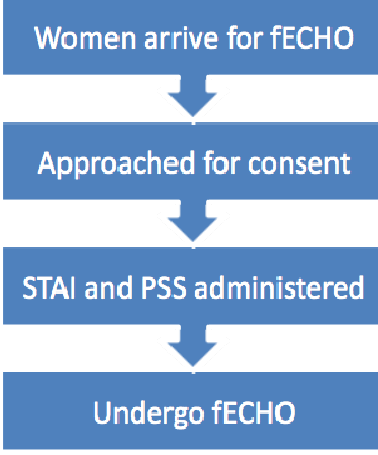

Group 2: Post-fECHO

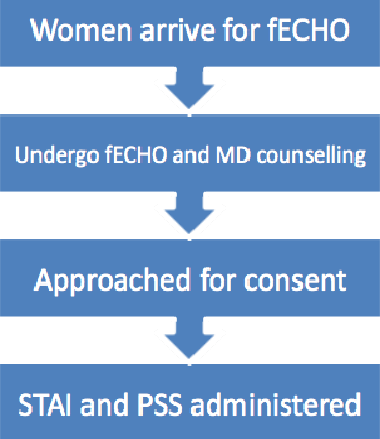

Figure 1. Study design. Legend: $\mathrm{fECHO}=$ fetal echocardiogram, STAI $=$ Speilberger State-Trait Inventory, PSS = Pregnancy Stress Scale. 
analyses to the normal post-fECHO group, and subsequently compared anxiety scores between pre-fECHO, normal post-fECHO and abnormal post-fECHO groups in order to show how the results of the fECHO influenced maternal anxiety. Scheduling conflicts prevented us from approaching 35 subjects, 26 were ineligible because they could not read English, and 12 did not wish to participate.

Participant characteristics are shown in Table 1. There were no significant differences in maternal age, gestational age or race between groups, however the prefECHO group had a higher incidence of prior miscarriage than the post-fECHO group. Self-reported indications for referral for fECHO did not differ significantly between the pre- and post-fECHO groups (Table 2) and subject baseline characteristics, including history of miscarriage, were not associated with state and trait scores (Table 3).

Table 1. Baseline subject characteristics.

\begin{tabular}{|c|c|c|c|}
\hline Variable & $\begin{array}{l}\text { Pre-fECHO N }=40 \\
N(\%) \text { or Mean } \pm \text { SD }\end{array}$ & $\begin{array}{l}\text { Post-fECHO N }=44 \\
\mathrm{~N}(\%) \text { or Mean } \pm \mathrm{SD}\end{array}$ & $\mathrm{p}$ value \\
\hline Age (years) & $30.7 \pm 6.9$ & $30.3 \pm 7.3$ & 0.82 \\
\hline Gestational age (weeks) & $23.4 \pm 5.1$ & $24.1 \pm 5.7$ & 0.57 \\
\hline \multicolumn{4}{|l|}{ Race } \\
\hline Caucasian & $26(65.0)$ & $18(40.9)$ & \\
\hline Hispanic & $7(17.5)$ & $16(36.3)$ & 0.178 \\
\hline Black or African-American & $3(7.5)$ & $5(11.4)$ & \\
\hline Asian & 0 & $4(9.1)$ & \\
\hline Other & $4(10)$ & $1(2.3)$ & \\
\hline \multicolumn{4}{|l|}{ Number of Pregnancies } \\
\hline Primigravid & $10(25)$ & $15(34.1)$ & 0.363 \\
\hline Multiparous & $30(75)$ & $29(65.9)$ & 0.361 \\
\hline \multicolumn{4}{|l|}{ Previous Miscarriage } \\
\hline Yes & $17(42.5)$ & $7(16)$ & 0.007 \\
\hline No & $23(57.5)$ & $37(84)$ & \\
\hline \multicolumn{4}{|l|}{ Maternal Medical problem } \\
\hline Yes & $12(30)$ & $13(30)^{*}$ & 0.964 \\
\hline No & $28(70)$ & $30(70)$ & \\
\hline \multicolumn{4}{|l|}{ Previous Therapist } \\
\hline Yes & $15(38)$ & $14(33)^{*}$ & 0.497 \\
\hline No & $24(62)$ & $29(67)$ & \\
\hline \multicolumn{4}{|l|}{ Diabetes } \\
\hline Yes & $7(17.5)$ & $4(90.9)$ & 0.254 \\
\hline No & $33(82.5)$ & $40(9.1)$ & \\
\hline \multicolumn{4}{|l|}{ Amniocentesis } \\
\hline Yes & $11(28)$ & $19(43.2)$ & 0.210 \\
\hline No & $28(72)$ & $25(56.8)$ & \\
\hline \multicolumn{4}{|l|}{ Previous fECHO } \\
\hline Yes & $2(5)$ & $5(11.4)$ & 0.292 \\
\hline No & $38(95)$ & $39(88.6)$ & \\
\hline
\end{tabular}

*Question was not answered by each subject.

Table 2. Self-reported indications for fECHO.

\begin{tabular}{|c|c|c|c|c|}
\hline Self-reported indication & Total cohort N (\%) & Pre-fECHO N (\%) & Post-fECHO N (\%) & p value \\
\hline Suspected anomaly on prior ultrasound & $49(58.3)$ & $24(60)$ & $25(56.8)^{*}$ & 0.768 \\
\hline Family history of congenital heart disease & $19(22.6)$ & $10(25)$ & $9(20.5)^{*}$ & 0.619 \\
\hline Maternal medical condition & $12(14.3)$ & $6(15)$ & $6(13.6)^{*}$ & 0.858 \\
\hline Abnormal amniocentesis & $6(7.1)$ & $3(7.5)$ & $3(6.8)^{*}$ & $1^{* *}$ \\
\hline Abnormal blood test & $2(2.4)$ & $1(2.5)$ & $1(2.3)$ & $1^{* *}$ \\
\hline High-risk (Mono-Di twins) & $2(2.4)$ & - & $2(4.5)$ & $0.5^{* *}$ \\
\hline Difficulty visualizing heart on prior ultrasounds (Obesity) & $1(1.2)$ & - & $1(2.3)$ & $1^{* *}$ \\
\hline Do not know & $6(7.1)$ & $4(10)$ & $2(4.5)$ & $0.42^{* *}$ \\
\hline
\end{tabular}

"Some subjects reported multiple indications; ${ }^{* *}$ Fisher's exact used for expected values with $\mathrm{n}<5$. 
Table 3. Univariable associations between baseline subject characteristics and state and trait scores among the entire cohort $(\mathrm{N}=84)$, pre-fECHO $(\mathrm{N}=40)$ and the post-fECHO cohorts $(\mathrm{N}=44)$.

\begin{tabular}{|c|c|c|c|c|c|c|c|c|c|}
\hline \multirow{2}{*}{$\begin{array}{l}\text { Variable } \\
\text { Maternal Age }\end{array}$} & \multicolumn{3}{|c|}{$\begin{array}{l}\text { Total cohort } \\
\mathrm{N}=84\end{array}$} & \multicolumn{3}{|c|}{$\begin{array}{c}\text { Pre-fECHO } \\
\mathrm{N}=40\end{array}$} & \multicolumn{3}{|c|}{$\begin{array}{c}\text { Post-fECHO } \\
\mathrm{N}=44\end{array}$} \\
\hline & Age $\geq 35 \mathrm{yr}$ & Age $<35 \mathrm{yr}$ & $\mathrm{p}$ value & Age $\geq 35 \mathrm{yr}$ & Age $<35 \mathrm{yr}$ & $\mathrm{p}$ value & Age $\geq 35 \mathrm{yr}$ & Age $<35 \mathrm{yr}$ & $\mathrm{p}$ value \\
\hline $\mathrm{N}(\%)$ & $26(31)$ & $58(69)$ & & $13(32.5)$ & $27(67.5)$ & & $13(29.5)$ & $31(70.5)$ & \\
\hline State Score Mean \pm SD & $38.3 \pm 15.2$ & $39.1 \pm 14$ & 0.81 & $42.6 \pm 17.3$ & $41.9 \pm 14.4$ & 0.88 & $33.9 \pm 12$ & $36.8 \pm 13.5$ & 0.51 \\
\hline Trait Score Mean \pm SD & $34.7 \pm 10.1$ & $35.6 \pm 10.9$ & 0.73 & $35.4 \pm 11.9$ & $34.4 \pm 10.4$ & 0.78 & $34 \pm 8.4$ & $36.6 \pm 11.4$ & 0.47 \\
\hline Parity & Primip & Multip & & Primip & Multip & & Primip & Multip & \\
\hline $\mathrm{N}(\%)$ & $25(29.8)$ & $59(70.2)$ & & $10(25)$ & $30(75)$ & & $15(34.1)$ & $29(65.9)$ & \\
\hline State Score Mean \pm SD & $37.1 \pm 11.7$ & $39.6 \pm 15.3$ & 0.46 & $42.0 \pm 11.0$ & $42.1 \pm 16.5$ & 0.98 & $33.8 \pm 11.4$ & $37.0 \pm 13.8$ & 0.44 \\
\hline Trait Score Mean \pm SD & $33.3 \pm 8.4$ & $36.1 \pm 11.4$ & 0.27 & $33.2 \pm 6.4$ & $35.2 \pm 11.9$ & 0.62 & $33.4 \pm 9.6$ & $37.0 \pm 11.0$ & 0.26 \\
\hline History of miscarriage & $\begin{array}{c}\text { Hx of } \\
\text { miscarriage }\end{array}$ & $\begin{array}{c}\text { No hx of } \\
\text { miscarriage }\end{array}$ & & $\begin{array}{c}\text { Hx of } \\
\text { miscarriage }\end{array}$ & $\begin{array}{c}\text { No hx of } \\
\text { miscarriage }\end{array}$ & & $\begin{array}{l}\text { Hx of } \\
\text { miscarriage }\end{array}$ & $\begin{array}{l}\text { No hx of } \\
\text { miscarriage }\end{array}$ & \\
\hline $\mathrm{N}(\%)$ & $24(28.6)$ & $60(71.4)$ & & $17(42.5)$ & $23(57.5)$ & & $7(16)$ & $37(84)$ & \\
\hline State Score Mean \pm SD & $42.3 \pm 14.7$ & $37.5 \pm 14.1$ & 0.17 & $45.1 \pm 14.3$ & $39.9 \pm 15.7$ & 0.28 & $35.4 \pm 14.3$ & $36.0 \pm 13.0$ & 0.91 \\
\hline Trait Score Mean \pm SD & $38.0 \pm 12.0$ & $34.2 \pm 9.9$ & 0.14 & $37.9 \pm 12.3$ & $32.3 \pm 9.0$ & 0.10 & $38.1 \pm 12.4$ & $35.4 \pm 10.3$ & 0.53 \\
\hline History of psychotherapy" & $\begin{array}{c}\text { Hx of } \\
\text { psychotherapy }\end{array}$ & $\begin{array}{c}\text { No hx of } \\
\text { psychotherapy }\end{array}$ & & $\begin{array}{c}\text { Hx of } \\
\text { psychotherapy }\end{array}$ & $\begin{array}{c}\text { No hx of } \\
\text { psychotherapy }\end{array}$ & & $\begin{array}{c}\text { Hx of } \\
\text { psychotherapy }\end{array}$ & $\begin{array}{c}\text { No hx of } \\
\text { psychotherapy }\end{array}$ & \\
\hline $\mathrm{N}(\%)$ & $29(34.5)$ & $53(63.1)$ & & $15(37.5)$ & $24(60)$ & & $14(32)$ & $29(66)$ & \\
\hline State Score Mean \pm SD & $39.2 \pm 14.2$ & $38.2 \pm 14.1$ & 0.76 & $45.5 \pm 13.6$ & $38.8 \pm 15.0$ & 0.17 & $32.5 \pm 11.7$ & $37.8 \pm 13.6$ & 0.22 \\
\hline Trait Score Mean \pm SD & $36.4 \pm 9.0$ & $33.7 \pm 10.2$ & 0.23 & $36.9 \pm 8.4$ & $31.8 \pm 9.2$ & 0.08 & $35.9 \pm 9.9$ & $35.3 \pm 10.8$ & 0.87 \\
\hline
\end{tabular}

*Question was not answered by each subject.

The mean state anxiety score of the pre-fECHO cohort $(42.1 \pm 15.1, \mathrm{~N}=40)$ was significantly higher than that of the normal post-fECHO group $(30.8 \pm 8.5, \mathrm{p}<0.001$, $\mathrm{N}=30$ ). There was no significant difference in trait scores (pre-fECHO $34.7 \pm 10.8$ vs normal post-fECHO $33.3 \pm 9.2, \mathrm{p}=0.56$ ). The difference in state anxiety score remained significant even when controlling for a history of miscarriage between the pre- and post-fECHO groups. Even when subjects who were counseled about an abnormal finding were included in the total postfECHO group $(\mathrm{N}=42)$, the difference in mean state anxiety score remained significant (pre-fECHO $42.1 \pm$ 15.1 vs total post-fECHO $35.9 \pm 13.0, p=0.05$ ).

To demonstrate how the results of the fECHO influenced maternal anxiety, we compared anxiety scores of subjects within the post-fECHO group (Table 4). State and trait scores were higher in the abnormal postfECHO group compared with the normal post-fECHO group (state $46.8 \pm 15.5$ vs $30.8 \pm 8.5, p=0.005$; trait $40.9 \pm 12.1$ vs $33.3 \pm 9.2, p=0.03)$. Anxiety levels of women awaiting $\mathrm{fECHO}$ and women who had already been told of an abnormal result were not statistically different (state pre-fECHO $42.1 \pm 15.1$ vs abnormal postfECHO $46.8 \pm 15.5, p=0.36$ ).

\section{DISCUSSION}

This study aimed to assess the impact of fECHO on maternal anxiety levels. In a prior study, we investigated differences in quantitative anxiety scores between women presenting for fECHO and a control group of pregnant women not presenting for fECHO [14]. We reported that the mean state anxiety score of the fECHO group (42.1 \pm 15.1) differed significantly from that of the control group $(32.8 \pm 11.3 ; \mathrm{p}=0.006)$ while there was no difference between the trait anxiety scores $(34.7 \pm 10.8$ vs $35.4 \pm$ $12.8 ; \mathrm{p}=0.8$ ). The results of this initial study led us to question if maternal anxiety changes after fECHO and physician counseling and if so, how. To our knowledge, our study is the first to compare maternal anxiety levels in groups of women prior to and following both normal and abnormal fECHO. Our study demonstrates that in women with normal fECHO results, maternal anxiety is low following the scan. In fact, the average state anxiety score in women following fECHO $(30.8 \pm 8.5)$ is lower than that reported in non-pregnant women and is no different from anxiety levels reported in pregnant women who had been screened for psychiatric co-morbidities (30.7 \pm 8.8$)$ [15]. While we would not go so far as to 
Table 4. (a) Univariate analysis: State and trait anxiety scores for total cohort and for pre-fECHO and post-fECHO cohorts; (b) Post-fECHO grouped according to result; (c) State and Trait scores for pre-fECHO group compared to abnormal post-fECHO group.

(a)

\begin{tabular}{lcccc}
\hline & Total cohort $(\mathbf{N}=\mathbf{8 4})$ & Pre-fECHO group $(\mathbf{N}=\mathbf{4 0})$ & ${\text { Total Post-fECHO group }(\mathbf{N}=\mathbf{4 2})^{*}}^{*}$ p value \\
\hline State score & $38.9 \pm 14.3$ & $42.1 \pm 15.1$ & $35.9 \pm 13$ & 0.048 \\
Trait score & $35.3 \pm 10.6$ & $34.7 \pm 10.8$ & $35.8 \pm 10.6$ & 0.63 \\
\hline
\end{tabular}

${ }^{*}$ Two subjects were excluded as they were counseled to follow-up in a few weeks for better visualization of the heart and could not be assigned to either the abnormal or normal groups.

(b)

\begin{tabular}{lccc}
\hline & Abnormal post-fECHO group $(\mathbf{N}=\mathbf{1 2})^{*}$ & Normal post-fECHO group (N = 30) & p value \\
\hline State score & $46.8 \pm 15.5$ & $30.8 \pm 8.5$ & 0.005 \\
Trait score & $40.9 \pm 12.1$ & $33.3 \pm 9.2$ & 0.03 \\
\hline
\end{tabular}

"Two subjects were excluded as they were counseled to follow-up in a few weeks for better visualization of the heart and could not be assigned to either the abnormal or normal groups.

(c)

\begin{tabular}{cccc}
\hline & Pre-fECHO group $(\mathbf{N}=\mathbf{4 0})$ & Abnormal Post-fECHO group (N = 12) & p value \\
\hline State score & $42.1 \pm 15.1$ & $46.8 \pm 15.5$ & 0.358 \\
Trait score & $34.7 \pm 10.8$ & $40.9 \pm 12.1$ & 0.95 \\
\hline
\end{tabular}

imply that undergoing a fECHO reduces maternal anxiety, we are nonetheless reassured by these findings and hypothesize that the anticipation of an abnormal result is what provokes anxiety.

As would be expected, anxiety levels remain high in women who receive an abnormal test result. However, what is remarkable is that the anxiety levels of women awaiting fECHO are no different quantitatively from anxiety levels of women who have been told by the pediatric cardiologist of an abnormality. This highlights just how anxiety-provoking referral for specialized testing is during pregnancy. Providers should be aware of how additional testing may affect the maternal psychological state and support should be made available to address maternal anxiety both prior to and following fECHO.

Changes in maternal anxiety in relation to timing and result of fECHO have been reported in the literature [18-21]. Björkhem et al. reported that a normal fECHO decreased anxiety among 65 families with a history of a prior child with congenital heart disease. There were no families with an abnormal fECHO in this study however. Sklansky et al. reported the results of a self-administered 7 -item questionnaire given to 213 women following fECHO; they found that $82 \%$ of women with a normal fECHO reported decreased anxiety, while only $28 \%$ of women with an abnormal fECHO reported decreased anxiety, and that maternal anxiety increased in only 5\% of the normal group and in $48 \%$ of the abnormal group. There are several distinctions between our study and that of Sklansky and colleagues. First, they did not administer the questionnaire in a standardized fashion and the actual timing of the maternal report relative to the $\mathrm{fECHO}$ results is unknown as women were instructed to take the questionnaires home. Second, anxiety was assessed qualitatively not quantitatively as it was in our study. Most importantly, theirs was a retrospective, within subject design in which all subjects received the questionnaires after the fECHO. The important question of anxiety experienced by women waiting for $\mathrm{fECHO}$ was not addressed. Interestingly, the Sklansky group reported that anxiety levels increased following an abnormal fECHO result, and in our study the anxiety scores did not differ between the pre-fECHO subjects and the postfECHO subjects who had an abnormality. The results of both studies share the important conclusion that a normal $\mathrm{fECHO}$ result is associated with comparatively less maternal anxiety.

Our study had certain limitations. First, the small sample size limited power to detect multiple associations. The STAI was offered only in English limiting generalizability to non-English speaking patients. Additionally, among the pre-fECHO group all subjects who were approached agreed to participate in the study, whereas in the postfECHO group 12 declined to participate. Refusal may have been due to time constraints as study participation in the post-fECHO subjects required that the patient remain in the hospital after the clinical visit to complete the questionnaires, whereas those in the pre-fECHO 
group completed the study while waiting for fECHO. However, of the 12 post-fECHO subjects who declined, 8 had an abnormal fECHO result raising the possibility of selection bias. Individual subjects were not tested repeatedly pre- and post-fECHO due to the concern of testretest recall bias. Finally, possible confounding variables, such as maternal education and income, were not included in the analysis as this data was not available in all subjects.

\section{CONCLUSION}

Maternal anxiety following healthy fECHO results is low. Women awaiting fECHO have high anxiety levels comparable to women who have just been told there is a fetal cardiac anomaly. The number of pregnant women who undergo fECHO each year is increasing due to widening medical criteria for referral and many of these women experience significant anxiety associated with this medical procedure. To carry out the mandate of integrated physical and mental health care, future studies are needed to evaluate the potential benefit of ongoing counseling and psychological support for maternal anxiety reduction both before and after fECHO.

\section{ACKNOWLEDGEMENTS}

We would like to thank our subjects for participating in this study as well as the clinic support staff that helped coordinate visits and interviews.

Dr. Williams reports support from Grant Number KL2 RR024157 from the National Centre for Research Resources, a component of the National Institutes of Health, and National Institutes of Health Roadmap for Medical Research. Its contents are solely the responsibility of the authors and do not necessarily represent the official view of National Centre for Research Resources or National Institutes of Health. Information on National Centre for Research Resources is available at http://www.ncrr.nih.gov/. Information on Re-engineering the Clinical Research Enterprise can be obtained from http://nihroadmap.nih.gov/clinicalresearch/overview-translational.asp.

\section{REFERENCES}

[1] Sahin, N.H. and Gungor, I. (2008) Congenital anomalies: Parents' anxiety and women's concerns before prenatal testing and women's opinions towards the risk factors. Journal of Clinical Nursing, 17, 827-836. doi:10.1111/j.1365-2702.2007.02023.x

[2] Leithner, K., Maar, A., Fischer-Kern, M., Hilger, E., Löf-fler-Stastka, H., et al. (2004) Affective state of women following a prenatal diagnosis: Predictors of a negative psychological outcome. Ultrasound in Obstetrics \& Gynecology, 23, 240-246. doi:10.1002/uog.978

[3] Filly, R.A. (2000) Obstetrical sonography: The best way to terrify a pregnant woman. Journal of Ultrasound in Medicine, 19, 1-5.
[4] Marteau, T.M., Cook, R., Kidd, J., Michie, S., Johnston, M. and Slack, J. (1992) The psychological effects of false-positive results in prenatal screening for fetal abnormality: A prospective study. Prenatal Diagnosis, 12, 205-214. doi:10.1002/pd.1970120309

[5] Marteau, T.M., Cook, R., Michie, S., Johnston, M., Slack, J. and Shaw, R.W. (1992) Psychological effects of having amniocentesis: Are these due to the procedure, the risk or the behaviour? Journal of Psychosomatic Research, 36, 395-402. doi:10.1016/0022-3999(92)90076-E

[6] Abuelo, D., Hopmann, M.R., Barsel-Bowers, G. and Goldstein, A. (1991) Anxiety in women with low maternal serum alpha-fetoprotein screening results. Prenatal Diagnosis, 11, 381-385. doi:10.1002/pd.1970110607

[7] Cristofalo, E.A., Dipietro, J.A., Costigan, K.A., Nelson, P. and Crino, J. (2006) Women's response to fetal choroid plexus cysts detected by prenatal ultrasound. Journal of Perinatalogy, 26, 215-223. doi:10.1038/sj.jp.7211489

[8] Diego, M.A., Jones, N.A., Field, T., Hernandez-Reif, M., Schanberg, S., Kuhn, C. and Gonzalez-Garcia, A. (2006) Maternal psychological distress, prenatal cortisol, and fetal weight. Psychosomatic Medicine, 68, 747-753. doi:10.1097/01.psy.0000238212.21598.7b

[9] Hedegaard, M., Henriksen, T.B., Sabroe, S. and Secher, N.J. (1993) Psychological distress in pregnancy and preterm delivery. British Medical Journal, 307, 934. doi:10.1136/bmj.307.6909.934-b

[10] Hedegaard, M., Henriksen, T.B., Secher, N.J., Hatch, M.C. and Sabroe, S. (1996) Do stressful life events affect duration of gestation and risk of preterm delivery? Epidemiology, 7, 337-338. doi:10.1097/00001648-199607000-00001

[11] Rondó, P.H., Ferreira, R.F., Nogueira, F., Ribeiro, M.C., Lobert, H. and Artes, R. (2003) Maternal psychological stress and distress as predictors of low birth weight, prematurity and intrauterine growth retardation. European Journal of Clinical Nutrition, 57, 266-272. doi:10.1038/sj.ejen.1601526

[12] Copper, R.L., Goldenberg, R.L., Das, A., Elder, N., Swain, M., Norman, G., et al. (1996) The preterm prediction study: Maternal stress is associated with spontaneous preterm birth at less than thirty-five weeks' gestation. American Journal of Obstetrics \& Gynecology, 175, 1286-1292. doi:10.1016/S0002-9378(96)70042-X

[13] Rychik, J., Ayres, N., Cuneo, B., Gotteiner, N., Hornberger, L., Spevak, P.J. and Van Der Veld, M. (2004) American Society of Echocardiography guidelines and standards for performance of the fetal echocardiogram. American Society of Echocardiography, 17, 803-810. doi:10.1016/j.echo.2004.04.011

[14] Rosenberg, K.B., Monk, C., Kleinman, C.S., Glickstein, J.S., Levasseur, S.M., Simpson, L.L., et al. (2010) Referral for fetal echocardiography is associated with increased maternal anxiety. American Journal of Obstetrics \& Gynecology, 31, 60-69. doi:10.3109/01674821003681472

[15] Spielberger, C.D. (1990) Manual for the state-trait anxiety inventory stai (form Y). Consulting Psychologists Press, Inc., Palo Alto. 
[16] Evans, L.M., Myers, M.M. and Monk, C. (2008) Pregnant women's cortisol is elevated with anxiety and depresssion-But only when comorbid. Archives of Women's Mental Health Arch Wom Ment Health, 11, 239-248. doi:10.1007/s00737-008-0019-4

[17] Field, T., Diego, M., Hernandez-Reif, M., Schanberg, S., Kuhn, C., Yando, R., et al. (2003) Pregnancy Anxiety and Comorbid Depression and anger: Effects on the fetus and neonate. Depress Anxiety, 17, 140-151. doi:10.1002/da. 10071

[18] Marteau, T.M., Johnston, M., Plenicar, M., Shaw, R.W. and Slack, J. (1988) Development of a self-administered questionnaire to measure women's knowledge of prenatal screening and diagnostic tests. Journal of Psychosomatic Research, 32, 403-408.

\section{doi:10.1016/0022-3999(88)90023-2}

[19] Fearn, J., Hibbard, B.M., Laurence, K.M., Roberts, A. and Robinson, J.O. (1982) Screening for neural-tube defects and maternal anxiety. British Journal of Obstetrics and Gynaecology, 89, 218-221. doi:10.1111/j.1471-0528.1982.tb03618.x

[20] Björkhem, G., Jörgensen, C. and Hanséus, K. (1997) Parental reactions to fetal echocardiography. Journal of Maternal-Fetal Medicine, 6, 87-92.

[21] Sklansky, M., Tang, A., Levy, D., Grossfeld, P., Kashani, I., Shaughnessy, R., et al. (2002) Maternal psychological impact of fetal echocardiography. Journal of the American Society of Echocardiography, 15, 159-166. doi: $10.1067 / \mathrm{mje} .2002 .116310$ 